\title{
Information and Announcements*
}

\section{Congratulations to the Newly Elected Members}

The Indian Academy of Sciences and Resonance: Journal of Science Education congratulate Professor Shobhana Narasimhan, Associate Editor (Resonance), Jawaharlal Nehru Centre for Advanced Scientific Research (JNCASR), Bengaluru, for being elected as International Honorary Member to the American Academy of Arts and Sciences.

Prof. Narasimhan has been honoured for her distinguished work in the field of sciences. She has made remarkable contributions to the rational design of nanomaterials, determining the effect of lowering of dimensionality and reduction of size on the properties of material.

We also extend our wishes to Professor Biman Bagchi from the Solid State and Structural Chemistry Unit of the Indian Institute of Science, Bengaluru, and Professor Kavita Singh, from the School of Arts and Aesthetics at the Jawaharlal Nehru University, Delhi, for being elected as the International Honorary Members to the American Academy of Arts and Sciences. 